\title{
Adaptive Agent Negotiation via Argumentation
}

\author{
Antonis Kakas \\ Dept. of Computer Science \\ University of Cyprus \\ 75 Kallipoleos Str. \\ 1678 Nicosia, Cyprus \\ +35722892706 \\ antonis@cs.ucy.ac.cy
}

\author{
Pavlos Moraitis \\ Dept. of Mathematics and Computer \\ Science \\ University René Descartes \\ 45 rue des Saints-Pères \\ 75270 Paris Cedex 6, France \\ $+33144553559$
}

pavlos@math-info.univ-paris5.fr

\begin{abstract}
In this paper, we study how argumentation can be used as a basis for negotiation between autonomous agents, where negotiation strategies of the different parties are represented as argumentation theories within their knowledge. We propose an argumentation based negotiation protocol in which offers by the negotiating parties are linked to different arguments that they can build according to their individual negotiation strategy. This protocol is able to take into account the different roles of agents and context of interaction, where the strength of the arguments supporting an offer can depend on these factors. The agents can adapt their negotiation strategies and offers, as their environment changes, in particular during the course of the negotiation as they exchange information. In addition, using abduction alongside with argumentation, agents can find negotiating conditions to support an argument for an offer, thus extending the negotiation object in order to help find an agreement. To illustrate further the advantages of the approach we also study how we can capture and extend negotiation strategies within another negotiation mechanism, in particular the well-known mechanism of bargaining with multiple parties. This exposes further the main advantages of modularity and flexibility of our argumentation based representation of negotiation strategies and the adaptability that this offers in a changing environment of negotiation.
\end{abstract}

\section{Categories and Subject Descriptors}

I.2.11 [Distributed Artificial Intelligence]: Multi-Agent Systems

\section{General Terms}

Theory

\section{Keywords}

Negotiation, Argumentation

\footnotetext{
Permission to make digital or hard copies of all or part of this work for personal or classroom use is granted without fee provided that copies are not made or distributed for profit or commercial advantage and that copies bear this notice and the full citation on the first page. To copy otherwise, or republish, to post on servers or to redistribute to lists, requires prior specific permission and/or a fee.

AAMAS’06, May 8-12, 2006, Hakodate, Hokkaido, Japan.

Copyright 2006 ACM 1-59593-303-4/06/0005...\$5.00.
}

\section{INTRODUCTION}

Negotiation is an important problem in multi-agent systems where autonomous agents need to be able to develop their own high-level strategy of operation. The diversity of strategies that different agents set can raise conflicts between them requiring a negotiation procedure to resolve these. Typically, negotiation aims to modify the local decisions of agents in order to avoid negative interactions and to emphasize the situations where positive or helpful interactions are possible.

The design of computational agents needs automated negotiation for various reasons [18]: 1) Agents interact in an open environment and may pursue different goals; 2) Several new applications such as electronic-business require an operational decision making level; and 3) The development of virtual enterprises requires negotiation and dynamic alliances. In all these cases, automated negotiation should provide a great help to save labor time in the sense that it may replace, at least partially, human negotiators. Automated negotiation has long been studied in the multi-agent systems field and different negotiation mechanisms have been proposed. These are based on game theoretic models (see e.g. [17]), heuristic approaches (see e.g. [6]) and some of them on argumentation (see e.g. [2, 14, 19]).

According to $[8,16]$ the approaches based on argumentation remove many of the limitations of the other approaches. These limitations stem from the facts that (a) the proposals made by the negotiating agents denote single points in the space of the negotiation agreements, (b) the only feedback that can be made to a proposal is a counter proposal, which itself is another point in the space, or an acceptance or withdrawal and (c) it is hard to change the set of issues under negotiation in the course of a negotiation. The added value of the approaches based on argumentation is that they provide additional information, over and above proposals. The arguments that agents can give to support their offers allow them to explicitly express their needs and rational of proposal. Agents have the possibility to question the arguments used by the other agents and to state their specific preferences for supporting their proposals. This gives the possibility to alter, during the negotiation process, the basic position of a negotiating party and its offers, e.g. by extending the negotiation space, or by offering treats or rewards in order to achieve an agreement. 
In this paper, we study negotiation using as a basis an argumentation framework $[9,10]$ that is able to express in a simple and direct way policies and negotiation strategies that can vary according to the particular circumstances in which these are applied. Based on this we propose a negotiation protocol where the decision making for negotiation is done through argumentative self-deliberation of the agents with their own theory. This is able to take into account the different roles that agents can have and specific contexts that describe particular or exceptional situations that agents could find themselves during the course of negotiation. Roles and context define in a natural way dynamic preferences on the decisions implied by the negotiation strategies of the agents as their environment (that includes the other negotiating agents) changes. In addition, aiming to provide agents with a level of robustness in the face of incomplete information from the negotiation environment, we integrate abduction within the argumentation framework. Agents are then able to deliberate on alternative choices and take decisions which are conditional on assumptions about the environment of the negotiation, e.g. assumptions on the preferences of other agents, the specific conditions in the current negotiation context, etc.

In order to illustrate further how argumentation can be used to enhance negotiation we will also study how we can capture and extend negotiation strategies within the traditional negotiation mechanism of bargaining with multiple parties. We show that their formulation within argumentation provides a representation that is more flexible in accommodating special or new requirements and allows us to enhance these negotiation strategies.

The rest of the paper is organized as follows. Section 2 presents briefly the background argumentation framework that we will use. Section 3 describes a new negotiation protocol based on argumentation. Section 4 develops a buying-selling scenario illustrating the main features of our protocol. It also studies representations of negotiation strategies within the negotiation mechanism of bargaining with multiple parties. Section 5 discusses related work and concludes.

\section{BACKGROUND}

\subsection{Argumentation with Dynamic Preferences}

In this section, we present the basic concepts of the argumentation framework on which our study of negotiation model will be based. This framework is presented in detail in [9, 10]. Each party in the negotiation will represent its own negotiation strategy (and background knowledge) within this framework.

Our aim is to use the declarative nature of the framework to allow the representation of flexible policies of negotiation strategies that can be sensitive to different circumstances as the negotiation process evolves. In particular, we will assume that negotiating agents are always associated with an (social) environment of interaction. We call this the context of interaction (i.e. market, enterprise, etc.). This determines the relationship between the possible roles the different agents can have within this environment and can influence the negotiation strategies used by the agents. Generally, roles are associated to a default context that defines shared social relations of different forms (e.g. authority, friendship, relationship, etc.) and specifies the interaction between different roles. For instance in the army context an officer gives orders that are obeyed by a soldier, or in the market a regular customer has benefits over a normal customer.

Consequently, a default context can be understood via priorities between the different roles. However, a default context determining the basic roles filled by the agents is not the only environment where they could interact and negotiate. For example two friends can also be colleagues, an officer and a soldier can be family friends in civil life. Therefore we consider a second level of context, called specific context, which can overturn the preimposed, by the default context, ordering between roles and establish a different relation between them. For instance, the authority relationship between an officer and a soldier would change under the specific context of a social meeting or regular and normal customers may have an equivalent consideration during the high season.

The argumentation framework that we will use was originally proposed in [11] and extended further in $[9,10]$ in order to deal with the dynamic nature of interaction of autonomous agents. It has been also adapted to model communication theories and dialogues (see e.g. [4, 12]). This framework uses a priority relation between arguments in the theory which is not a static relation but context dependent that captures the non-static preferences associated to roles and specific context of interaction. We define an agent's argumentation theory for describing a preference policy, e.g. a negotiation strategy, in an environment with roles and context as follows. The full presentation of the argumentation framework can be found in (e.g. [10]).

Definition 1. An agent's argumentative policy theory, $\mathbf{T}$, is a theory $\mathbf{T}=\left(\left(\mathcal{T}, \mathcal{T}_{o}\right) \mathscr{P}_{\mathrm{R}}, \mathscr{P}_{\mathrm{C}}\right)$ where $\mathcal{T}$ contains the argument rules in the form of definite Horn logic rules, $\mathscr{P}_{\mathrm{R}}$ contains priority rules which are also definite Horn rules with head h-p $\left(\mathrm{r}_{1}, \mathrm{r}_{2}\right)$, s.t. $\mathrm{r}_{1}, \mathrm{r}_{2} \in$ $\mathcal{T}$ (h-p standing for higher priority) and all rules in $\mathscr{P}_{\mathrm{C}}$ are also priority rules with head h-p $\left(\mathrm{R}_{1}, \mathrm{R}_{2}\right)$ s.t. $\mathrm{R}_{1}, \mathrm{R}_{2} \in \mathcal{P}_{\mathrm{R}} \cup \mathcal{P}_{\mathrm{C}}$. $\mathcal{T}_{\circ}$ contains auxiliary rules of the agent's background knowledge

We therefore have three levels in an agent's theory. In the first level we have the rules $\mathcal{T}$ that refer directly to the subject domain of the agent. We call these the Object-level Decision Rules of the agent. In the other two levels we have rules that relate to the policy under which the agent uses his object-level decision rules according to roles and context. We call the rules in $\mathscr{P}_{\mathrm{R}}$ and $\mathscr{P}_{\mathrm{C}}$, Role (or Default Context) Priorities and Specific Context Priorities respectively.

In a more general setting, we can associate the dynamic preferences expressed through the rules of the two later levels, to normal situations (related to a default context) and specific situations (related to specific contexts).

Definition 2. Let $(\mathcal{T}, \mathcal{P})$ be a theory, $\mathrm{T} \subseteq \mathcal{T}$ and $\mathrm{P} \subseteq \mathcal{P}$. Then $(\mathrm{T}, \mathrm{P})$ is admissible iff $(\mathrm{T} \cup \mathrm{P})$ is consistent and for any $\left(\mathrm{T}^{\prime}, \mathrm{P}^{\prime}\right)$ if $\left(\mathrm{T}^{\prime}, \mathrm{P}^{\prime}\right)$ attacks $(T, P)$ then $(T, P)$ attacks $\left(T^{\prime}, P^{\prime}\right)$.

We remind that the notion of attack between arguments in a theory is based on the possible conflicts between a literal L and its negation and on the priority relation given by $h-p$ in the theory. 
Given a ground literal L then L is a credulous (respectively skeptical) consequence of the theory iff $L$ holds in a (respectively every) maximal, w.r.t. set inclusion, admissible subset of the theory.

Hence for an object-level argument (from $\mathcal{T}$ ) to be admissible it needs to take along with its priority arguments (from $\mathscr{P}$ where $\mathscr{P}=$ $\left.\mathscr{P}_{\mathrm{R}} \cup \mathscr{P}_{\mathrm{C}}\right)$ to make itself at least "as strong" as the opposing counter-arguments. This need for priority rules can repeat itself when the initially chosen ones can themselves be attacked by opposing priority rules and again we would need to make now the priority rules themselves at least as strong as their opposing ones.

In several cases the admissibility of an argument depends on whether we have or not some background information about the specific case in which we are reasoning. Sometimes this information maybe just unknown and agents can reason further to find assumptions related to the unknown information under which they can build an admissible argument. We can formalize this conditional form of argumentative reasoning by defining the notion of supporting information and extending argumentation with abduction on this missing information. This, as we will see later, is very useful for negotiation.

Definition 3. Let $\mathrm{T}=\left(\left(\mathcal{T}_{0}, \mathcal{T}\right), \mathcal{P}\right)$ be a theory, and, $\mathcal{A}$, a distinguished set of predicates in the language of the theory, called abducible predicates ${ }^{1}$. Given a goal $\mathrm{G}$, a set $\mathrm{S}$ of ground abducible literals consistent with the non-defeasible part of the background theory $\mathcal{T}_{o}$, is called a strong (respectively weak) supporting information for $\mathrm{G}$ iff $\mathrm{G}$ is a skeptical (respectively credulous) consequence of $\left(\left(\mathcal{T}_{\circ} \cup \mathrm{S}, \mathcal{T}\right), \mathcal{P}\right)$ and $\mathrm{S}$ is a minimal (w.r.t. set inclusion) such set that has this property.

An argument can then be generalized as follows.

Definition 4. Let $\mathrm{T}=\left(\left(\mathcal{T}_{0}, \mathcal{T}\right), \mathcal{P}\right)$ be a theory, and $\mathcal{A}$ its abducible predicates. A supported argument in $\mathrm{T}$ is a tuple $(\Delta, \mathrm{S})$, where $\mathrm{S}$ is a set of abducible literals consistent with $\mathcal{T}_{0}$ and $\Delta$ is a set of argument rules, which is not admissible in $\left(\left(\mathcal{T}_{0}, \mathcal{T}\right), \mathcal{P}\right)$, but is admissible in the theory $\left(\left(\mathcal{T}_{0} \cup \mathrm{S}, \mathcal{T}\right), \mathscr{P}\right)$. We say that $\mathrm{S}$ supports the argument $\Delta$ and that $\Delta$ is conditional on $\mathrm{S}$.

The supporting information refers to the incomplete and evolving information of the external environment of interaction. Typically, this information pertains to the context of the environment, the roles between agents or any other dynamic aspect of the agent's knowledge.

Given the above framework the argumentative deliberation of an agent can be formalized via the following basic reasoning functions that will be useful in our study of negotiation.

Definition 5. Let $\mathrm{Ag}$ be an agent, T his argumentation theory, G a goal and $\mathrm{S}$ a set of abducibles consistent with the background part, $\mathcal{T}_{o}$, of its theory. Then we say that $A g$ deliberates on $\mathrm{G}$, under $\mathrm{S}$, to produce $\mathrm{s}^{\mathrm{ag}}$, denoted by deliberate $\left(A g, \mathrm{G}, \mathrm{S} ; \mathrm{s}^{\mathrm{ag}}\right)$, iff

${ }^{1}$ For simplicity, we will assume that abducible predicates are a subset of the background predicates.
$S^{a g}$ is a strong supporting information for $\mathrm{G}$ in the theory $\mathrm{T}$ and $S^{a g}=S^{a g}-S$. If $S^{a g}$ is a subset of $S$ then we say that $A g$ accepts $G$ under $\mathrm{T}$ and $\mathrm{S}$ and is denoted by $\operatorname{accept}(\mathbf{A g}, \mathbf{G}, \mathbf{S})$.

\section{THE NEGOTIATON PROTOCOL}

In this section, we will set up a negotiation protocol for intelligent agents by exploiting the internal argumentative deliberation that each agent can perform. The negotiation object(s), e.g. the price of a product, decided by the involved agents are associated to a class of goals for each agent, e.g. sell at high prices for a seller and buy at low prices for a buyer. During the negotiation the agents accumulate extra information about the external environment via supporting information conditions that each agent generates to support, in its own theory, the argument for its offer (see definition 3). This supporting information is then associated with the offer and in some cases it can be seen as extending the negotiation object. The various items of supporting information are collected together and build gradually during the negotiation allowing thus a form of incremental deliberation of the agents as they acquire more information. We note that in this paper, we are not interested in the communication protocol supporting the negotiation. The main elements of the negotiation protocol we propose are as follows:

1. Each agent insists in making proposals corresponding to its own high-level goal as long as its deliberation within its theory and the accumulated supporting information (agreed by the two agents so far) produces new supporting information (see definitions 3, 4) for this goal. A proposal contains an offer corresponding to the negotiation object (e.g. a specific price), together with supporting information representing conditions under which this offer is made. Different offers will typically have different supporting information, e.g. a goal to buy at a low price may contain several prices as possible offers, each of them being supported by the appropriate information. Thus the negotiation object can be extended and may cover several issues related to the initial issue, e.g. terms and conditions under which an agent could accept a specific price.

2. The first of the two negotiating agents who is unable to produce a new offer with supporting information for his goal, abandons it and searches for supporting information, if any, under which it can accept the (counter) proposal of the other agent. For example, a seller agent unable to find another way to support offers with high prices considers selling at a low price and looks for supporting information under which it may be able to do so.

3. In such a case, the negotiation enters a conciliation phase and if the receiver agent can endorse the proposed supporting information the negotiation ends with agreement on this offer and the supporting information accumulated so far. Otherwise, the sender takes this into account and tries again to find another way to support the goal (offer) of the other agent. If this is not possible, then the negotiation ends in failure.

Besides the argumentative functions deliberate and accept (see definition 5) we use three other functions of an agent, external to his argumentative reasoning, that are needed in the negotiation process. The function propose(Offer, $\left.e_{j}, s_{i}\right)$ is used by a sender agent to determine what information to send to the other agent: Offer is an offer drawn from an agent's goal and proposed 
as an alternative on which negotiating agents could agree; $e_{j}$ is the evaluation (see below) by the sender of the supporting information $s_{j}$ sent to it in the previous step by the other agent; and $s_{i}$ is the new supporting information produced by the deliberation of the sender for its Offer.

The function evaluate $\left(\mathrm{Ag}, \mathrm{s}_{i}\right)$ produces an evaluation $e_{i}$, where for each (abducible) literal in the supporting information $s_{i}$ this is either accepted or rejected (i.e. negated) according to some process of evaluation by a receiving agent $\mathrm{Ag}$. This can therefore be considered as a critique (see e.g. [16]) of $s_{i}$ by Ag. This function can vary in complexity from a simple check in the agent's database to an argumentative deliberation on $s_{i}$ and a new subsidiary negotiation process on this.

The function update(S,e) updates, through an external mechanism, the store of accumulated supporting information $S$ with the new information $e$ produced by the agent's evaluation of the supporting information sent by the other agent. Part of the task of this function is also to maintain the consistency of the store $S$. We require that conflicts are resolved in favour of the latest information i.e. in favour of the information in $e$. In this paper we will not be concerned with the details of this mechanism as they are not important for the specification of the protocol. Thus $S$ is related to the concept of commitment store (see [7, 13]) adopted by the agent literature (see e.g. [1]). A commitment store holds all the statements to which an agent has committed during the negotiation. In our case $S$ corresponds to the union of the commitment stores and contains all the additional information produced in the course of the negotiation and accepted by each negotiating agent.

The negotiation protocol we are proposing operates via the alternate application by each one of the two negotiating agents of the algorithm given below. This defines formally the steps of the negotiation process. Note that in the presentation of this algorithm, we assume that it is agent $\mathrm{X}$ who is applying it. Agent $\mathrm{Y}$ applies exactly the same algorithm, and therefore the reader has only to replace $\mathrm{X}$ with $\mathrm{Y}$ in order to see how a negotiation process between two agents would evolve (see Section 4 for a buyingselling scenario).

Below we will denote by $\mathrm{G}^{\mathrm{X}}$ the argumentation goal corresponding to an offer $\mathrm{O}^{\mathrm{X}}$ and by $\mathrm{e}_{\mathrm{n}}^{\mathrm{X} \rightarrow \mathrm{Y}}$ the evaluation by agent $\mathrm{X}$ of the supporting information sent by agent $\mathrm{Y}$. Also we will denote by $\mathrm{s}^{\mathrm{I}, \mathrm{J}}$ the supporting information generated by agent, I, for the goal of agent, J, (I, J can take the values X or Y).

Each agent (agent $\mathrm{X}$ here) can receive three types of proposals. In the first one, denoted by $\operatorname{propose}\left(O^{Y}, e^{Y \rightarrow X}, s_{n}^{Y, Y}\right)$, another agent, $\mathrm{Y}$, sends to $\mathrm{X}$ an offer, $\mathrm{O}^{\mathrm{Y}}$, with a new alternative supporting information, $s_{n}{ }_{n}, Y$. This proposal also includes, $e^{Y \rightarrow X}$ (empty in the initial proposal), which is the evaluation by $\mathrm{Y}$ of the supporting information sent to it in the previous step by $\mathrm{X}$ for its offer $\mathrm{O}^{\mathrm{X}}$. In the second type of proposal, denoted by propose $\left(O^{Y}\right.$, $e_{m}{ }^{Y \rightarrow X}, \otimes$ ), the other agent, $\mathrm{Y}$, sends to $\mathrm{X}$ (via step 4.4 for agent $\mathrm{Y})$ its answer to an offer made previously by $\mathrm{X}$ to consider the offer $\mathrm{O}^{\mathrm{Y}}$ of $\mathrm{Y}$, i.e. $\mathrm{X}$ has entered the conciliation phase and $\mathrm{Y}$ is responding to this. This answer contains the evaluation, $e_{n}{ }^{Y} \rightarrow X$, by $\mathrm{Y}$ of the terms (or conditions) proposed by $\mathrm{X}$ in order to accept an offer $\mathrm{O}^{\mathrm{Y}}$ satisfying the goal of $\mathrm{Y}$. Finally, in the third type of proposal, denoted by $\operatorname{propose}\left(O^{X}, \otimes, s_{n}{ }^{Y, X}\right)$ another agent, Y, sends to $\mathrm{X}$ an answer where it proposes the conditions, $\mathrm{s}_{\mathrm{n}} \mathrm{Y}, \mathrm{X}$, under which it could accept the offer $\mathrm{O}^{\mathrm{X}}$ of $\mathrm{X}$, i.e. $\mathrm{Y}$ has previously entered the conciliation phase.

Begin

$\mathrm{S}=\{\} ; \mathrm{n}=0$

1. agent $\mathrm{X}$ receives a proposal $\mathrm{O}$ from an agent $\mathrm{Y}$

2. if $\mathrm{O}$ is of the form propose $\left(\mathrm{O}^{\mathrm{Y}}, \mathrm{e}_{\mathrm{n}-1}^{\mathrm{Y} \rightarrow \mathrm{X}}, \mathrm{s}_{\mathrm{n}}^{\mathrm{Y}}{ }^{\mathrm{Y}}\right)$ then

2.1. $\mathrm{e}_{\mathrm{n}}^{\mathrm{X} \rightarrow \mathrm{Y}} \leftarrow$ evaluate $\left(\mathrm{X}, \mathrm{s}_{\mathrm{n}}{ }^{\mathrm{Y}, \mathrm{Y}}\right)$;

2.2. $\mathrm{S} \leftarrow$ update $\left(\mathrm{S}, \mathrm{e}_{\mathrm{n}-1} \mathrm{Y} \rightarrow \mathrm{X} \cup \mathrm{e}_{\mathrm{n}}^{\mathrm{X} \rightarrow \mathrm{Y}}\right)$

2.3. if $e_{n}^{X \rightarrow Y}=s_{n}^{Y, Y}$ and $\operatorname{accept}\left(X, G^{Y}, S\right)$ then END(agreement, $\mathrm{O}^{\mathrm{Y}}$ )

else

2.4. $\mathrm{n} \leftarrow \mathrm{n}+1$; find $\mathrm{s}_{\mathrm{n}}{ }^{\mathrm{X}, \mathrm{X}}$ s.t. deliberate $\left(\mathrm{X}, \mathrm{G}^{\mathrm{X}}, \mathrm{S} ; \mathrm{s}_{\mathrm{n}}{ }^{\mathrm{X}, \mathrm{X}}\right)$ and $\mathrm{s}_{\mathrm{n}}{ }^{\mathrm{X}, \mathrm{X}} \neq \mathrm{s}_{\mathrm{i}}{ }^{\mathrm{X}, \mathrm{X}} \forall \mathrm{i}<\mathrm{n}$

2.5 if $\mathrm{s}_{\mathrm{n}}{ }^{\mathrm{X}}$ exists then propose $\left(\mathrm{O}^{\mathrm{X}}, \mathrm{e}_{\mathrm{n}-1}^{\mathrm{X} \rightarrow \mathrm{Y}}, \mathrm{s}_{\mathrm{n}}^{\mathrm{X}, \mathrm{X}}\right)$ to $\mathrm{Y}$ else (Enter Conciliation Phase) $\mathrm{m}=0$

2.6. $\quad \mathrm{S} \leftarrow$ update $\left(\mathrm{S}, \mathrm{e}_{\mathrm{n}}^{\mathrm{Y} \rightarrow \mathrm{X}}\right)$

2.7. $\mathrm{m} \leftarrow \mathrm{m}+1$; find $\mathrm{s}_{\mathrm{m}} \mathrm{X}, \mathrm{Y}$ s.t. deliberate $\left(\mathrm{X}, \mathrm{G}^{\mathrm{Y}}, \mathrm{S} ; \mathrm{s}_{\mathrm{m}}{ }^{\mathrm{X}, \mathrm{Y}}\right)$ and $\mathrm{s}_{\mathrm{m}}^{\mathrm{X}, \mathrm{Y}} \neq \mathrm{s}_{\mathrm{j}}^{\mathrm{X}, \mathrm{Y}} \forall \mathrm{j}<\mathrm{m}$

2.8. if $\mathrm{s}_{\mathrm{m}}{ }^{\mathrm{X}} \mathrm{Y}$ exists then $\operatorname{propose}\left(\mathrm{O}^{\mathrm{Y}}, \otimes, \mathrm{s}_{\mathrm{m}}^{\mathrm{X}, \mathrm{Y}}\right)$ to $\mathrm{Y}$

2.9. $\quad$ endif

2.10. endif

2.11. endif

2.12. endif

3. if $\mathrm{O}$ is of the form propose $\left(\mathrm{O}^{\mathrm{Y}}, \mathrm{e}_{\mathrm{m}}^{\mathrm{Y} \rightarrow \mathrm{X}}, \otimes\right)$ then

3.1. goto 2.6 ; endif

4. if $\mathrm{O}$ is of the form propose $\left(\mathrm{O}^{\mathrm{X}}, \otimes, \mathrm{s}_{\mathrm{k}}^{\mathrm{Y}, \mathrm{X}}\right)$ then

4.1. $\mathrm{e}_{\mathrm{k}}^{\mathrm{X} \rightarrow \mathrm{Y}} \leftarrow$ evaluate $\left(\mathrm{X}, \mathrm{s}_{\mathrm{k}}^{\mathrm{Y}, \mathrm{X}}\right)$

4.2. $\mathrm{S} \leftarrow$ update $\left(\mathrm{S}, \mathrm{e}_{\mathrm{k}}^{\mathrm{X} \rightarrow \mathrm{Y}}\right)$

4.3. if $\mathrm{e}_{\mathrm{k}}^{\mathrm{X} \rightarrow \mathrm{Y}}=\mathrm{s}_{\mathrm{k}}{ }_{\mathrm{Y}, \mathrm{X}}$ and $\operatorname{accept}\left(\mathrm{X}, \mathrm{G}^{\mathrm{X}}, \mathrm{S}\right)$ then

$\operatorname{END}\left(\right.$ agreement, $\mathrm{O}^{\mathrm{X}}$ )

4.4. else propose $\left(\mathrm{O}^{\mathrm{X}}, \mathrm{e}_{\mathrm{k}}^{\mathrm{X} \rightarrow \mathrm{Y}}, \otimes\right)$

4.5. endif

4.6. endif

end

We can show the following formal properties for the above negotiation protocol.

Proposition 1. Let $X, Y$ be two agents and $T_{X}, T_{Y}$ their respective argumentation policy theories which contain a finite number of sentences. Then,

(1) any negotiation process will terminate.

(2) if a negotiation process terminates with agreement on an offer $O$ and $S$ is the final set of supporting information accumulated during the negotiation then the corresponding goal, $G(O)$, is a skeptical conclusion of both $T_{X} \cup S$ and $T_{Y} \cup S$. 


\section{Proof}

(1) Termination follows directly from the fact that, when an agent deliberates again, either on its own goal or the goal of the other agent, the algorithm requires that it produces new supporting information and the fact, that there can only be a finite number of different supporting information generated by the theories of the agents.

(2) There are two cases for the process to terminate in agreement. In the first case (step 2.3) agent $\mathrm{X}$ agrees on the offer $\mathrm{G}^{\mathrm{Y}}$ of the other agent (agent $Y$ ) when $\operatorname{accept}\left(X, G^{Y}, S\right)$ holds. By the definition of $\operatorname{accept}\left(X, G^{Y}, S\right)$ this means that $G^{Y}$ is a skeptical conclusion of $T_{X} \cup S$. This is also a skeptical conclusion of $T_{Y} \cup S$ since agent $Y$ has produced this offer for its own goal $G^{Y}$ (at its step 2.4) and its supporting information has been accepted by agent $X$ and updated in $S$ at step 2.2. Similarly, we show that the result holds also in the second case where agreement can be reached, at step 4.3.

\section{EXAMPLES OF NEGOTIATON}

In this section, we will present two examples of negotiation in order to illustrate how our argumentation-based approach to negotiation works in practice. First we will present a bargaining example of buying-selling transactions that illustrates how the specific negotiation protocol that we have proposed in the previous section applies and how the argumentative deliberation of the agents is performed within this protocol. Then we will investigate how our argumentation framework can be used for representing and extending negotiation strategies within another negotiation mechanism, in particular the well-known mechanism of bargaining with multiple parties.

\subsection{A Buying-Selling Scenario}

Let us consider a buying-selling scenario between a seller agent, called $\mathrm{X}$, who has the goal, $\mathrm{G}^{\mathrm{X}}$, to sell a product at a high price and a buyer agent, called Y, who has the (opposing) goal, $\mathrm{G}^{\mathrm{Y}}$, to buy this product at a low price. These goals generate corresponding offers and counter-offers containing different values for the negotiation object, namely the price for the product. Therefore agents are trying to find an agreement on a specific price by agreeing either on an offered selling price $\mathrm{O}^{\mathrm{X}}$ or on an offered buying price $\mathrm{O}^{\mathrm{Y}}$. We assume that the seller has the following simplified (where only two prices high and low exist) argumentation policy for selling products. We present only a part of this theory ${ }^{2}$.

\footnotetext{
${ }^{2}$ Here and below we will use Logic Programming notation where any term starting with a capital letter represents a variable. Abusing this notation we will denote the constant names of the priority rules in the role part, $\mathcal{P}_{\mathrm{R}}$, of the theory with capital $\mathrm{R}$ letters (subscripted) and the priority rules in the specific context part, $\mathcal{P}_{\mathrm{C}}$, of the theory with subscripted capital $\mathrm{C}$ letters. All variables are universally quantified over the whole rule in which they appear. Each rule represents the collection of ground rules that we obtain by grounding its variables over the Herbrand universe of the theory.
}

The goal $\mathrm{G}^{\mathrm{X}}$ of the seller is $\mathrm{G}^{\mathrm{X}}=$ sell(prd,buyer,high $\_$price). There are two ways or methods to get this "high_price", either through a normal payment method or through payment via installements. The object-level theory $\mathcal{T}^{\mathrm{X}}$ contains the rules:

$\mathrm{r}_{1}$ : sell(Prd, Ag, high_price, method 1$) \leftarrow$ pay_normal(Ag,Prd)

$\mathrm{r}_{2}$ : sell(Prd, Ag, high_price, method2) $\leftarrow$ pay_install(Ag,Prd)

$\mathrm{r}_{3}$ : sell(Prd, Ag, low_price $) \leftarrow$ pay_cash $($ Ag,Prd $)$

$\mathrm{r}_{4}:$ sell $($ Prd, Ag, Price $) \leftarrow$ sell $($ Prd, Ag, Price, Method $)$

$\mathrm{r}_{5}: \neg$ sell $\left(\mathrm{Prd}, \mathrm{Ag}, \mathrm{P}_{2}\right) \leftarrow \operatorname{sell}\left(\mathrm{Prd}, \mathrm{Ag}, \mathrm{P}_{1}\right), \mathrm{P}_{2} \neq \mathrm{P}_{1}$

$\mathrm{r}_{6}: \neg \operatorname{sell}\left(\operatorname{Prd}, \mathrm{Ag}, \mathrm{P}, \mathrm{M}_{2}\right) \leftarrow \operatorname{sell}\left(\operatorname{Prd}, \mathrm{Ag}, \mathrm{P}, \mathrm{M}_{1}\right), \mathrm{M}_{2} \neq \mathrm{M}_{1}$

Its role and context priority theories, $\mathscr{P}_{\mathrm{R}}^{\mathrm{X}}$ and ${\mathscr{P}^{\mathrm{X}}}_{\mathrm{C}}$, are given below. They contain the policy of the seller under which it should treat or negotiation with the various types of customers. For example, it should prefer to sell with normal paying conditions (e.g. cash or visa) over payment by installments when the buyer is a normal customer (see $\mathrm{R}_{1}$ ). Also that there is always a preference to sell at high price (see $\mathrm{R}_{2}, \mathrm{R}_{3}$ ) but for regular customers there are conditions under which the seller would sell at low price (see $\mathrm{R}_{4}, \mathrm{R}_{5}$ ). This low price offer to a regular customer applies during a sales season (see $\mathrm{C}_{3}, \mathrm{C}_{4}$ ) and not during a high season (see $\mathrm{C}_{1}$, $\mathrm{C}_{2}$ ) where the preference of a high price is stronger.

$\mathrm{R}_{1}: h-p\left(r_{1}(\operatorname{Prd}, A), r_{2}(\operatorname{Prd}, A)\right) \leftarrow \operatorname{normal}(A)$
$\mathrm{R}_{2}: h-p\left(r_{1}(\operatorname{Prd}, A), r_{3}(\operatorname{Prd}, A)\right)$
$R_{3}: h-p\left(r_{2}(\operatorname{Prd}, A), r_{3}(\operatorname{Prd}, A)\right)$
$R_{4}: h-p\left(r_{3}(\operatorname{Prd}, A), r_{1}(\operatorname{Prd}, A)\right) \leftarrow \operatorname{regular}(A)$, buy_2$(A, \operatorname{Prd})$
$R_{5}: h-p\left(r_{3}(\operatorname{Prd}, A), r_{1}(\operatorname{Prd}, A)\right) \leftarrow \operatorname{regular}(A)$, late_del $(A, \operatorname{Prd})$

$\mathrm{C}_{1}: \mathrm{h}-\mathrm{p}\left(\mathrm{R}_{2}(\operatorname{Prd}, \mathrm{A}), \mathrm{R}_{4}(\operatorname{Prd}, \mathrm{A})\right) \leftarrow$ $\mathrm{C}_{2}: \mathrm{h}-\mathrm{p}\left(\mathrm{R}_{2}(\operatorname{Prd}, A), \mathrm{R}_{5}(\operatorname{Prd}, A)\right) \leftarrow$ $\mathrm{C}_{3}: \mathrm{h}-\mathrm{p}\left(\mathrm{R}_{4}(\operatorname{Prd}, \mathrm{A}), \mathrm{R}_{2}(\operatorname{Prd}, \mathrm{A})\right) \leftarrow$ $\mathrm{C}_{4}: \mathrm{h}-\mathrm{p}\left(\mathrm{R}_{5}(\operatorname{Prd}, \mathrm{A}), \mathrm{R}_{2}(\operatorname{Prd}, \mathrm{A})\right) \leftarrow$ $\mathrm{C}_{5}$ : h-p $\left(\mathrm{R}_{4}(\operatorname{Prd}, A), \mathrm{R}_{5}(\operatorname{Prd}, A)\right)$

high_season

high_season

sales_season

sales_season

Lets us consider the particular negotiation scenario given below as it evolves through the application of our proposed negotiation protocol and study how the seller uses its own argumentative reasoning, with the above theory, in this scenario. For simplicity of presentation we will show the update function simply as union. We would also like to note that in the following example we only present the reasoning made by each agent during the negotiation process. The presentation of the communication protocol (e.g. structure of exchanged locutions, performatives used, etc.) implementing this negotiation dialogue is out of the scope of this paper. This is part of our future work and it will be based on the work we proposed in $[4,12]$.

Seller X (step 1)

-propose(high_price, \{\}$, \mathrm{s}_{0} \mathrm{X}, \mathrm{X}$ )

$-\mathrm{S}_{0} \mathrm{X}, \mathrm{X}=\{$ pay_normal, normal $\left.(\mathrm{Y})\}\right)$

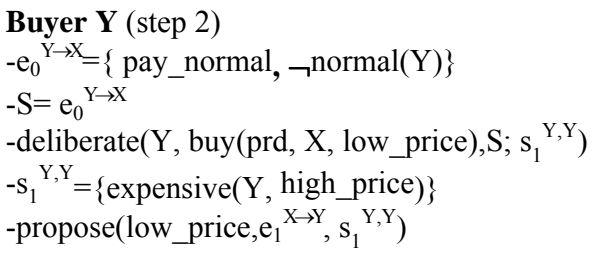


Seller X (step 3)

$-\mathrm{e}_{1}^{\mathrm{X} \rightarrow \mathrm{Y}}=\mathrm{s}_{1} \mathrm{Y}, \mathrm{Y}$

$-\mathrm{S} \leftarrow \mathrm{S} \cup \mathrm{e}_{1} \mathrm{X} \rightarrow \mathrm{Y}$

-Cannot Accept

-deliberate(X, sell(prd, Y, high_price), $S ; \mathrm{s}_{1}{ }^{\mathrm{X}, \mathrm{X}}$ )

$-\mathrm{s}_{1} \mathrm{X}, \mathrm{X}=\{$ pay_install $\}$

-propose(high_price, $\mathrm{e}_{1}^{\mathrm{X} \rightarrow \mathrm{Y}}, \mathrm{s}_{1}^{\mathrm{X}, \mathrm{X}}$ )

Buyer Y (step 4)

$-\mathrm{e}_{2}^{\mathrm{Y} \rightarrow \mathrm{X}}=\neg \mathrm{S}_{1} \mathrm{X}, \mathrm{X}$

$-\mathrm{S} \leftarrow \mathrm{S} \cup \mathrm{e}_{2}{ }^{\mathrm{Y} \rightarrow \mathrm{X}}$

-deliberate(Y, buy(prd, X, low_price), $\mathrm{S} ; \mathrm{s}_{2}{ }^{\mathrm{Y}, \mathrm{Y}}$ )

$-\mathrm{s}_{2}{ }^{\mathrm{Y}, \mathrm{Y}}=\{$ pay_cash $\}$

-propose(low_price, $\mathrm{e}_{2}^{\mathrm{Y} \rightarrow \mathrm{X}}, \mathrm{s}_{2}{ }^{\mathrm{Y}, \mathrm{Y}}$ )

Seller X (step 5)

$-\mathrm{e}_{3}{ }^{\mathrm{X} \rightarrow \mathrm{Y}}=\mathrm{S}_{2} \mathrm{Y}, \mathrm{Y}$

$-\mathrm{S} \leftarrow \mathrm{S} \cup \mathrm{e}_{3} \mathrm{X} \rightarrow \mathrm{Y}$

-Cannot Accept

-deliberate(X, sell(prd, Y, high_price), S; s)

-fails to find new $s$.

Seller X (step 6): X Enters Conciliation Phase -deliberate(X, sell(prd,Y,low_price), $S ; \mathrm{s}_{1}{ }^{\mathrm{X}, \mathrm{Y}}$ )

$-\mathrm{s}_{1}^{\mathrm{X}, \mathrm{Y}}=\{\operatorname{regular}(\mathrm{Y})$, buy_2(Y,prd) $\left.\}\right)$

-propose (low_price, $\otimes, \mathrm{s}_{1} \mathrm{X}, \mathrm{Y}$ )

Buyer Y (step 7)

$-\mathrm{e}_{4}{ }^{\mathrm{Y} \rightarrow \mathrm{X}}=\{\operatorname{regular}(\mathrm{Y}), \neg$ buy $2(\mathrm{Y}, \operatorname{prd})\}$

-propose(low_price, $\left.\mathrm{e}_{4}{ }^{\mathrm{Y} \rightarrow \overline{\mathrm{X}}}, \otimes\right)$

Seller X (step 8)

$-\mathrm{S} \leftarrow \mathrm{S} \cup \mathrm{e}_{4}{ }^{\mathrm{Y} \rightarrow \mathrm{X}}$

-deliberate(X, sell(prd, Y, low_price), S; $\mathrm{s}_{2}{ }^{\mathrm{X}, \mathrm{Y}}$ )

$-\mathrm{s}_{2}{ }^{\mathrm{X}, \mathrm{Y}}=\{$ later_del(Y,prd $\left.\left.)\right\}\right)$

-propose (low_price, $\otimes, \mathrm{s}_{2}{ }^{\mathrm{X}, \mathrm{Y}}$ )

Buyer Y (step 9)

$-\mathrm{e}_{5}^{\mathrm{Y} \rightarrow \mathrm{X}}=\mathrm{s}_{2} \mathrm{X}, \mathrm{Y}$

$\mathrm{S} \leftarrow \mathrm{S} \cup \mathrm{e}_{5}{ }^{\mathrm{Y} \rightarrow \mathrm{X}}$

-accept offer; END(agreement, low_price)

At step 1 the seller agent proposes to the buyer agent to buy the product at a high price (which is the preferred price for the seller according to his theory). Step 2 of the example corresponds to the second step of the negotiation algorithm. At this stage the buyer agent cannot accept the offer. He thus deliberates on his own goal and proposes a low price. At step 3 the seller cannot accept the proposal for low price: his theory even under the fact that this is not a normal customer contains admissible arguments for the contrary of this proposal to sell low. He now needs to find a new argument for his goal of selling high. Deliberating on his goal, he finds such an argument, using the object-level rule $r_{2}$. This new argument needs the support (or is conditional on) pay_install(buyer, prd) and the seller offers this information to the buyer insisting on his offer of a high_price.
At step 5 the seller deliberates again on his own goal (to sell high) but cannot find a new solution anymore. It therefore initiates at step 6 a conciliation phase of the negotiation where he considers the offer made by the buyer, i.e. to sell at low_price. He deliberates on this goal and finds (an admissible argument using $r_{3}$ and $\mathrm{R}_{4}$ ) that it is possible to do so if the customer can pay cash (which is already in the store $\mathrm{S}$ ), is a regular one and he accepts to buy two of the products. In this way the seller agent extends the negotiation object by submitting for negotiation these latter two conditions. Note that for this to skeptically derive the offer to sell low, we need to be at a sales season and that the context $\mathrm{C}_{1}$ rule must not apply i.e. that we are not in high season, facts that the seller knows in its background theory. The buyer confirms that he is a regular one but refuses to buy two (i.e. it generates a critique to the extended offer). The seller then has another solution to sell low to a regular customer conditional on late delivery, which is accepted by the buyer agent and the process ends in agreement on "low price with a late delivery".

Note that the late delivery condition has been generated dynamically in the particular scenario extending the negotiation object of the price in order to reach agreement. In some other scenario it could happen that this will not enter at all the process.

As we have already remarked, the evaluation function evaluate $\left(\mathrm{Ag}, \mathrm{s}_{\mathrm{i}}\right)$, can take, in its more complex case the form of a new subsidiary argumentative deliberation of $A g$ on $s_{i}$. This could lead to a nested negotiation process, where the negotiation object is extended further and covers additional issues related to the original (or parent) object. Let us illustrate this subsidiary argumentative deliberation, along with the possible nested negotiation steps, by considering that the buyer agent in the above example has the following argumentation theory for his general policy with regards to methods of payment and how he uses this to evaluate proposals from the seller.

$$
\begin{aligned}
& \mathrm{r}_{1}: \text { pay }(\operatorname{Prd}, \text { Price, install }) \leftarrow \text { reasonable }(\text { Prd, Price }) \\
& \mathrm{r}_{2}: \text { pay }(\operatorname{Prd}, \operatorname{Price}, \text { cash }) \leftarrow \neg \text { expensive }(\operatorname{Prd}, \text { Price }) \\
& \mathrm{r}_{3}: \neg \text { pay }\left(\operatorname{Prd}, \mathrm{P}, \mathrm{M}_{2}\right) \leftarrow \text { pay }\left(\operatorname{Prd}, \mathrm{P}, \mathrm{M}_{1}\right), \mathrm{M}_{2} \neq \mathrm{M}_{1} \\
& \mathrm{R}_{1}: \mathrm{h}-\mathrm{p}\left(\mathrm{r}_{1}(\operatorname{Prd}, \mathrm{P}), \mathrm{r}_{2}(\operatorname{Prd}, \mathrm{P})\right) \\
& \mathrm{R}_{2}: \mathrm{h}-\mathrm{p}\left(\mathrm{r}_{2}\left(\operatorname{Prd}, \mathrm{P}_{1}\right), \mathrm{r}_{1}\left(\operatorname{Prd}, \mathrm{P}_{2}\right)\right) \leftarrow \neg \text { cash_flow_problems, } \mathrm{P}_{1} \leq \mathrm{P}_{2} \\
& \text { cash_bonus }(\operatorname{Prd}, \text { Bonus }) \\
& \mathrm{C}_{1}: \mathrm{h}-\mathrm{p}\left(\mathrm{R}_{2}\left(\operatorname{Prd}, \mathrm{P}_{1}, \mathrm{P}_{2}\right), \mathrm{R}_{1}\left(\operatorname{Prd}, \mathrm{P}_{2}\right)\right) \leftarrow \text { discount }\left(\operatorname{Prd}, \mathrm{P}_{1}, \mathrm{D}\right), \mathrm{D}>30 \%
\end{aligned}
$$

According to this policy buyer prefers to pay by installments rather than cash. When he has no cash flow problems and there is a cash bonus he prefers to pay in cash (provided also that the cash price is not higher than the installments price). In the specific case where the price is at a discount of more than $30 \%$ then this preference to pay cash is stronger than the preference to pay by installments.

At the fourth step of the example above the buyer evaluates the proposal of the seller to pay by installments via an argumentative deliberation on pay(prd, high_price,install). The negotiation object is extended from <Price $>$ to $<$ Price, MethodOfPayment $>$. Despite the fact that high_price is a reasonable price for the buyer pay(prd,high_price,install) it is not a skeptical conclusion of his theory as he has an admissible argument for pay(prd, low_price, install). The buyer would then make this counter offer (in a new subsidiary step) to pay by installments but at a low price. If the seller rejects this offer the buyer could then re-evaluate and, assuming that there is a cash 
bonus at a discount of over $30 \%$ for low_price, the above theory of the buyer would then give the skeptical conclusion pay(prd, low_price, cash). Hence the buyer would then offer to pay cash for a low price.

\subsection{Bargaining with Multiple Parties}

In a bargaining setting, the negotiation process starts with an initial offer formulated by one of the participating parties and then proceeds with an alternate exchange of offers. This process stops either when an agreement is found or one of the involved parties decides to terminate the negotiation with a specific party (or all the parties) without any agreement. Normally, in many realistic scenarios a member participates in several bargaining processes simultaneously, e.g. a buyer contacts several merchants in search for the best terms for buying a given product.

We will study an example type of bargaining considered in [5]. According to this scenario an agent is responsible for negotiating a price with several potential sellers on behalf of a buyer. The offers sent by the sellers are arranged in a queue and then are taken from the queue and evaluated. A buyer agent can accept an offer, propose a counter-offer or terminate the negotiation with the corresponding party. If the offer is accepted, the negotiating agent asks the other party to confirm the agreement, and upon receiving the confirmation, he terminates all the negotiation processes in which he is involved. In the contrary case where a confirmation refusal message arrives or the confirmation message is not received after an agreed delay, an occurrence of the event "no_confirmation" is generated and the agent continues to negotiate with the other remaining parties. In the case where there are no more potential sellers remaining, the overall negotiation process is terminated with a failure.

Below we will show how this evaluation strategy of the buyer agent for each offer it receives can be captured in a natural and direct way within our argumentation framework ${ }^{3}$. We will use the term "offer" to designate the prices proposed by the sellers, and the term "counter-offer" to designate the prices proposed by the buyer. We will also use the following predicates and constants in the representation of the buyer's theory:

- $\operatorname{offer}(P, X)$ : the current offer of the party $\mathrm{P}$ is $\mathrm{X}$

- first_offer $(P, X)$ : indicates the first offer $\mathrm{X}$ of the party $\mathrm{P}$

- previous_offer $(P, X)$ : the previous offer of the party $\mathrm{P}$ was $\mathrm{X}$

- counter_offer $(P, X): \mathrm{X}$ is a counter offer suggested as a reply to $P$ 's most recent offer reception

- previous_counter_offer $(P, X): X$ is a counter offer suggested as a reply to the $P$ 's second most recent offer reception

- $\operatorname{accept}(P, X)$ : indicates that the most recent offer $X$ of $P$ can be accepted

- terminate $(P)$ : indicates that the negotiation with the party $\mathrm{P}$ must be terminated

- good_price: indicates a price that can be accepted

- increment: indicates an acceptable amount of raising the price

- acceptable_price: it is the top price that can be accepted

- best_offer $(X): \mathrm{X}$ is the current best offer

${ }^{3}$ Note that we will not be concerned here with the issue of how the buyer selects the next offer to be evaluated. This can be done using another modularly separated preference policy written analogously in the theory of the buyer.
- time_remaining $(T)$ : indicates the time before the deadline of the bargaining

In the theory that we will present here we will also extend the standard bargaining scenario in order to illustrate further the flexibility of our direct and modular representation of the problem. For example, we can allow for a price greater to "good_price" to be acceptable under some specific circumstances, e.g. when we have only a few remaining sellers or little time remaining before the deadline of the bargaining, provided that the buyer has a big urgency for buying a specific product.

We will represent one by one the policy rules of the evaluation strategy of the buyer directly from their natural specification. Note that "counter_offer", "accept" and "terminate" are all contrary to each other, i.e. each one implies the negation of all others. Similarly, different prices for "accept" and "counter_offer" are contrary to each other.

"If the first offer of P is greater than the "good_price" then ask for the "good_price"."

$\mathrm{r}_{1}$ :counter_offer $\left(\mathrm{P}, \mathrm{good} \_\right.$price $) \leftarrow$ first_offer $(\mathrm{P}, \mathrm{X}), \mathrm{X}>$ good_price.

"If $\mathrm{P}$ has not improved his offer terminate the negotiation with him."

$\mathrm{r}_{2}$ : terminate $(\mathrm{P}) \leftarrow$ offer $(\mathrm{P}, \mathrm{Y})$, previous_offer $(\mathrm{P}, \mathrm{Z}), \mathrm{Y} \geq \mathrm{Z}$.

"If $\mathrm{P}$ has improved his offer with respect to the previous one, then concede a small amount with respect to the previous counter-offer but do not counter-propose a price below the current best offer." $\mathrm{r}_{3}$ : counter_offer $(\mathrm{P}, \min (\mathrm{X}+$ increment, $\mathrm{W})) \leftarrow \quad$ offer $(\mathrm{P}, \mathrm{Y})$, previous_offer $(\mathrm{P}, \mathrm{Z}), \mathrm{Y}<\mathrm{Z}$, previous_counter_offer(P,X), best_offer(W)

"If the offer of $\mathrm{P}$ is below the acceptable price and it is the best one that has been received then you can accept it"

$\mathrm{r}_{4}: \quad \operatorname{accept}(\mathrm{P}, \mathrm{Y}) \quad \leftarrow$ best_offer(Z), offer(P,Y), $\mathrm{Y} \leq \mathrm{Z}, \quad \mathrm{Y} \leq$ acceptable_price

"If somebody offers the "good_price" then this offer can be accepted."

$\mathrm{r}_{5}: \operatorname{accept}(\mathrm{P}, \mathrm{Y}) \leftarrow \operatorname{offer}(\mathrm{P}, \mathrm{Y}), \mathrm{Y} \leq$ good_price

"A good price has preference over any other response and over other prices below the acceptable price"

$\mathrm{R}_{1}: \mathrm{h}-\mathrm{p}\left(\mathrm{r}_{5}(\mathrm{P}, \mathrm{Y}), \mathrm{r}_{2}(\mathrm{P})\right)$

$\mathrm{R}_{2}$ : h-p $\left(\mathrm{r}_{5}(\mathrm{P}, \mathrm{Y}), \mathrm{r}_{3}(\mathrm{P}, \mathrm{Z})\right)$

$\mathrm{R}_{3}: \mathrm{h}-\mathrm{p}\left(\mathrm{r}_{5}(\mathrm{P}, \mathrm{Y}), \mathrm{r}_{4}(\mathrm{P}, \mathrm{Y} 1)\right) \leftarrow \mathrm{Y}<\mathrm{Y} 1$

"Prefer to accept a price below the acceptable price over terminating the process"

$\mathrm{R}_{4}: \mathrm{h}-\mathrm{p}\left(\mathrm{r}_{4}(\mathrm{P}, \mathrm{Y}), \mathrm{r}_{2}(\mathrm{P})\right)$

"Prefer to counter offer asking for a better price over accepting a price below the acceptable price"

$\mathrm{R}_{5}: \mathrm{h}-\mathrm{p}\left(\mathrm{r}_{3}(\mathrm{P}, \mathrm{H}), \mathrm{r}_{4}(\mathrm{P}, \mathrm{Y})\right)$

"When we are in a critical situation, i.e. there are only a few sellers left and time is running out, then prefer to accept an offer over making a counter offer. This applies only when there is a big urgency to acquire the product." 
$\mathrm{R}_{6}: \mathrm{h}-\mathrm{p}\left(\mathrm{r}_{4}(\mathrm{P}, \mathrm{Y}), \mathrm{r}_{3}(\mathrm{P}, \mathrm{H})\right) \leftarrow$ few_sellers_remaining, time_remaining $(\mathrm{T}), \mathrm{T} \leq$ time threshold

$\mathrm{C}_{1}: \mathrm{h}-\mathrm{p}\left(\mathrm{R}_{5}(\mathrm{P}, \mathrm{H}), \mathrm{R}_{6}(\mathrm{P}, \mathrm{Y})\right)$

$\mathrm{C}_{2}: \mathrm{h}-\mathrm{p}\left(\mathrm{R}_{6}(\mathrm{P}, \mathrm{Y}), \mathrm{R}_{5}(\mathrm{P}, \mathrm{H})\right) \leftarrow$ big_urgency

\section{RELATED WORK AND CONCLUSIONS}

We have presented an argumentation-based negotiation framework, which like other approaches that use argumentation (see e.g. [8, 16]), removes many of the limitations of the alternative approaches. Our approach confirms the advantages of an argumentation-based approach to negotiation and covers the requirements defined in [8] for an effective argumentation-based negotiation. Comparing with other argumentation-based approaches (see e.g. [2, 14, 19]), the integration of roles (or default context) and contexts (or specific contexts) within the deliberation process of the agents facilitates agents to dynamically adapt their strategies, offers and counter-offers as the environment of negotiation changes. In addition, integrating abduction within the argumentative reasoning of the agents we allow them to extend, during the process of negotiation, the negotiation object with other objects pertaining to conditions under which an agent could accept an offer. In most current approaches (see e.g. [14]), agents generate proposals and counter-proposals until an agreement or a failure is reached, by reasoning only on their own goals or objectives. In our framework we have proposed a negotiation protocol where an agent, when he cannot satisfy his own goal, can consider in a conciliation phase, the other agent's goal and search for conditions under which he could accept it. This can lead again to the extension of the negotiation object helping to reach an agreement. We have shown that our argumentation framework is well suited for modeling this negotiation protocol. However, the negotiation protocol itself, is not crucially dependent on the specific underlying logical framework for argumentation and it could be built similarly on top of other such frameworks (e.g. [3, 15]) provided also that they are extended with abduction so that they can have supported arguments. We also have investigated through the extension of a case study, originally presented in [5], the application of our argumentation framework for representing negotiation strategies within another negotiation mechanism, in particular the wellknown mechanism of bargaining with multiple parties. Other negotiation problems, e.g. the resource allocation problem, can also be captured and extended in the same way. This framework and the presented examples have been implemented using the Gorgias [20] system.

Our future work concerns the introduction of multi-criteria techniques from decision theory in the evaluation, by the agents, of the exchanged offers and counter-offers and the development of an associated communication protocol to support the negotiation protocol, based on the work we proposed in $[4,12]$. We wish also to investigate how to integrate within the same framework a "personality" component for each one of the negotiating agents, expressed also as an argumentation theory, and study how this can influence the negotiation process between different types (e.g. cooperative or self-interested) of agents.

\section{REFERENCES}

[1] Amgoud L. and Parsons S. Agent dialogues with conflicting preferences, in Proc. ATAL'01, pp. 190-205, 2001.
[2] Amgoud L., Parsons S., and Maudet N. Arguments, dialogue and negotiation, in Proc. ECAI'00, pp. 338-342, 2000.

[3] Brewka, G. and Eiter, T. Preferred Answer Sets for Extended Logic Programs, Artificial Intelligence 109, 297-356, 1999.

[4] Dimopoulos, Y., Kakas, A., and Moraitis, P. Argumentation Based Modeling of Embedded Agent Dialogues, in ArgMAS'05, Utrecht, The Netherlands, 2005.

[5] Dumas M., Governatori G., ter Hofstede, A.H.M., and Oaks, P. "A formal approach to negotiating agents development", in Electronic Commerce and Applications, Vol. 1, No 2, Elsevier Science Publications, 2002.

[6] Faratin, P., Sierra, C., and Jennings, N.R. "Using similarity criteria to make issue trade-offs in automated negotiation", in Artificial Intelligence 142 (2) 205-237, 2001.

[7] Hamblin, C.L. Fallacies, Methuen, London, 1970.

[8] Jennings, N. R., Faratin, P., Lomuscio, A.R., Parsons, S., Sierra C., and Wooldridge, M. Automated Negotiation: Prospects, methods and challenges, in J. of Group Decision and Negotiation 10 (2) 199-215, 2001.

[9] Kakas A. and Moraitis, P. Argumentative Agent Deliberation, Roles and Context, in Electronic Notes in Theoretical Computer Science, (ENTCS), 70(5), 2002.

[10] Kakas, A. and Moraitis, P. Argumentation based decision making for autonomous agents", in Proc. AAMAS'03, pp. 883-890, 2003.

[11] Kakas, A., Mancarella P., and Dung, P.M. The acceptability semantics for logic programs, in Proc. ICLP'94, pp. 504519, 1994.

[12] Kakas, A., Maudet, N., and Moraitis, P. Modular Representation of Agent Interaction Rules through Argumentation, in J. of AAMAS, Springer, vol. 11, no. 2, pp. 189-206, 2005.

[13] MacKenzie, J. Question-begging in non-cumulative systems, in Journal of Philosophical Logic, 8:117-133, 1979.

[14] Parsons, S., Sierra, C., and Jennings, N.R. Agents that reason and negotiate by arguing, in Journal of Logic and Computation, 8(3), pp. 261-292, 1998.

[15] Prakken, H. and Sartor, G. A dialectical model of assessing conflicting arguments in legal reasoning, in Journal of Artificial Intelligence and Law, Vol. 4, pp. 331-368, 1996.

[16] Rahwan, I., Ramchurn, S. D., Jennings, N. R., McBurney, P., Parsons S., and Sonenberg, L. Argumentation-based negotiation, in Knowledge Engineering Review, 18 (4) 343$375,2003$.

[17] Rosenschein, J.S. and Zlotkin, G. Roles of Encounter, MIT Press, 1994.

[18] Sandholm, T.W. Distributed Rational Decision Making, In Multiagent Systems: A modern approach to distributed artificial intelligence, G. Weiss (eds.), pp. 201-258, 1999.

[19] Sycara, K. Argumentation: planning other agents' plans, in Proc. IJCAI'89, pp. 517-523, 1989.

[20] Gorgias: Argumentation with Abduction, http://www.cs.ucy.ac.cy/ nkd/gorgias 